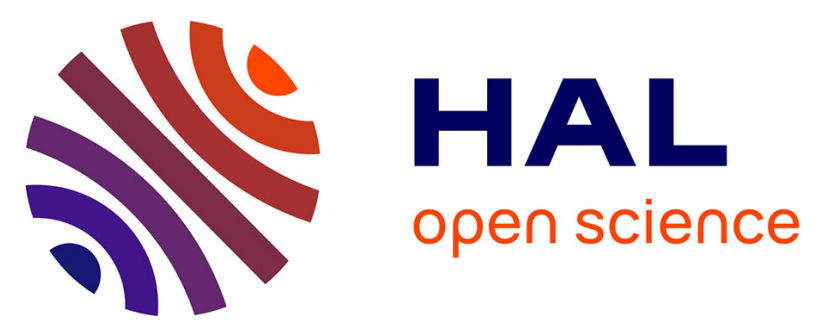

\title{
Integrated active mixing and biosensing using low frequency vibrating mixer and Love-wave sensor for real time detection of antibody binding event
}

F. Kardous, L. El Fissi, J.-M. Friedt, F. Bastien, W. Boireau, R. Yahiaoui, J.-F. Manceau, S. Ballandras

\section{To cite this version:}

F. Kardous, L. El Fissi, J.-M. Friedt, F. Bastien, W. Boireau, et al.. Integrated active mixing and biosensing using low frequency vibrating mixer and Love-wave sensor for real time detection of antibody binding event. Journal of Applied Physics, 2011, 109 (9), pp.094701. 10.1063/1.3576113 . hal-00611524

\author{
HAL Id: hal-00611524 \\ https://hal.science/hal-00611524
}

Submitted on 24 Mar 2021

HAL is a multi-disciplinary open access archive for the deposit and dissemination of scientific research documents, whether they are published or not. The documents may come from teaching and research institutions in France or abroad, or from public or private research centers.
L'archive ouverte pluridisciplinaire HAL, est destinée au dépôt et à la diffusion de documents scientifiques de niveau recherche, publiés ou non, émanant des établissements d'enseignement et de recherche français ou étrangers, des laboratoires publics ou privés. 


\title{
Integrated active mixing and biosensing using low frequency vibrating mixer and Love-wave sensor for real time detection of antibody binding event
}

\author{
F. Kardous, ${ }^{1, a)}$ L. El Fissi, ${ }^{2, b)}$ J-M Friedt, ${ }^{3, c)}$ F. Bastien, ${ }^{1}$ W. Boireau, ${ }^{1}$ R. Yahiaoui, ${ }^{1}$ \\ J.-F. Manceau, ${ }^{1}$ and S. Ballandras ${ }^{1,3}$ \\ ${ }^{1}$ FEMTO-ST, University de Franche-Comté, CNRS, ENSMM, UTBM F-25044 Besançon, France \\ ${ }^{2}$ ICTEAM, ELEC - Place du Levant 3 à 1348 Louvain-la-Neuve, Belgium \\ ${ }^{3}$ SENSeOR, 32 Avenue de l'Observatoire, 25044 Besançon, France
}

(Received 12 January 2011; accepted 12 March 2011; published online 5 May 2011)

\begin{abstract}
The development of lab-on-chip devices is expected to dramatically change biochemical analyses, allowing for a notable increase of processing quality and throughput, provided the induced chemical reactions are well controlled. In this work, we investigate the impact of local acoustic mixing to promote or accelerate such biochemical reactions, such as antibody grafting on activated surfaces. During microarray building, the spotting mode leads to low efficiency in the ligand grafting and heterogeneities which limits its performances. To improve the transfer rate, we induce a hydrodynamic flow in the spotted droplet to disrupt the steady state during antibody grafting. To prove that acoustic mixing increases the antibody transfer rate to the biochip surface, we have used a Love-wave sensor allowing for real-time monitoring of the biological reaction for different operating conditions (with or without mixing). An analysis of the impact of the proposed mixing on grafting kinetics is proposed and finally checked in the case of antibody-antigen combination. (C) 2011 American Institute of Physics. [doi:10.1063/1.3576113]
\end{abstract}

\section{INTRODUCTION}

Although numerous work has been focused on the development of lab-on-chip devices for improving bio-chemical analysis, there is still great interest in investigating new solutions for accurate detection and reaction monitoring, particularly by using guided acoustic waves because of their remarkable sensitivity and stability. ${ }^{1-3}$

This study focuses on the analysis of biochemical reaction kinetics measured using direct detection biosensors. The two most common transducers in the field of direct detection biosensors are based on the conversion of an adsorbed mass to an electrical velocity through the interaction with an acoustic wave (quartz crystal resonators and surface acoustic wave sensors) ${ }^{4-6}$ or with an evanescent electromagnetic wave (surface plasmon resonance). ${ }^{7-11}$ Besides the difference in the interaction mechanism, most of these devices allow for monitoring the adsorption kinetics as the solution stands still in an open well configuration, with some significant developments in the area of packaging toward the continuous flow of the reagents, which appears to be significant challenge for acoustic sensors. ${ }^{12-14}$ One significant exception to this approach is Biacore's surface plasmon resonance (SPR) system in which the reagents flow in a microfluidic channel. ${ }^{15,16}$ The SPR system is equipped with a microfluidic cartridge, allowing for the dynamic circulation of fluid and thus dynamic transfer of biological molecules to a biochip surface. Nevertheless, in the case of low Reynolds number hydrodynamic flows, and particularly in the case of a high Damkohler number, reactions with the active surface are

\footnotetext{
${ }^{a)}$ Electronic mail: faten.kardous@femto-st.fr.

b)Electronic mail: lamia.elfissi@uclouvain.be.

${ }^{c)}$ Electronic mail: jmfriedt@femto-st.fr.
}

mainly governed by diffusive effects. ${ }^{17}$ Therefore, the reaction and sensing performances may be limited considering time scales and sensitivity.

An alternate solution to continuous fluid flow is local mixing of the liquid layer over the sensing surface. Mixing at the microfluidic level is a well known challenging issue since sub-millimeter dimensions and a low Reynolds number usually yields laminar flows. ${ }^{18,19}$ Some works propose to induce active mixing by an additional energy source plugged into the system to create flow instabilities. For example, ultrasonic mixers using stationary wave patterns or surface acoustic waves (SAW) were developed in order to decrease the mixing time and to improve the homogeneity of continuous-flow mixtures. ${ }^{20}$ Some microdevices using microchannels even allow usto generate microdrops of reagents and to coalesce them in a carrier continuous phase. $^{21}$

Furthermore, an alternative approach to continuous delivery microfluidic systems is the handling of discrete droplets. The electrowetting-based linear-array droplet mixer, for example, proves that microdroplets can be transported, merged, and actively mixed using an electrostatic field. ${ }^{22}$ An acoustic field can also be used for that purpose; several examples have been presented using, for the most part, high frequency vibrations such as SAW devices. ${ }^{23}$ Pioneering work proves the feasibility of a system composed of a droplet based SPR system coupled to a SAW microfluidic platform. ${ }^{24}$ This enabled Renaudin and co-workers to experiment with a device incorporating SPR sensing and a SAW actuation onto a common substrate ${ }^{25}$ instead of two independent systems investigated by Galopin. ${ }^{24}$ However, they observed that SAW action causes a parasitic SPR response due to a thermal effect, in addition to the improved surface coverage during the antibody immobilization reaction. 
In this paper, we propose a fully acoustic solution combining a Love-wave sensor and a low frequency vibrating mixer with the aim of a real-time monitoring of biological reactions while they are excited by an active mixing, independent of the SPR system. Within this framework, we wish to assess that mixing increases the antibodies $(\mathrm{Ab})$ transfer to a sensing area surface and increases the reaction kinetics by removing the dependency with the protein diffusion coefficient in a liquid, while inducing minimum disturbance to the sensing capability of the Love mode transducer due to its low thermal coefficient and differential measurement strategy. We believe that this approach will be the beginning of a real time detection of the binding effect in a spotting mode, which until now, is still considered as a black box.

In the first section, we describe our sensor and mixing system, detailing their structural and operational features and the experimental preparation. We then report our experimental results on the acoustically stimulated adsorption. We finally propose an analysis of the impact of acoustic mixing on the adsorption process to explain the observed kinetics using the Love-wave sensor.

\section{MATERIALS AND METHODS}

\section{A. Surface acoustic wave sensor}

Love-wave devices consist of delay lines built on a (AT, Z) cut of quartz for minimizing the temperature drift coefficient around room temperature. The wave guidance is achieved by depositing a $2.5 \mu \mathrm{m}$ thick silica layer at the top of an AT-cut of quartz. The thickness was selected as a trade-off between an acceptable gravimetric sensitivity and a reasonably simple and reproducible sensor fabrication (PECVD silica layer deposition duration). The Love-wave is excited and detected using inter-digital transducers (IDTs) composed of 50 pairs of 4-finger-per-wavelength electrodes made of $200 \mathrm{~nm}$ thick aluminum strips deposited by evaporation and patterned using a lift-off process. The grating period is $10 \mu \mathrm{m}$, i.e., a wavelength close to $40 \mu \mathrm{m}$, yielding an operation frequency in the vicinity of $125 \mathrm{MHz}$. A $3.2 \mathrm{~mm}$ long cavity is achieved between the two delay line IDTs, corresponding to the location where biochemical reactions are assumed to take place (the so-called sensing area). This area was covered by successive evaporation and lift-off of $10 \mathrm{~nm}$ of titanium and $50 \mathrm{~nm}$ of gold. The acoustic aperture is 3.5 $\mathrm{mm}$ (about 90 wavelengths). The silica overlays used here as guiding layers are patterned to access the bonding pads. ${ }^{26}$ Figure 1 shows a scheme of the delay line and Fig. 2 shows a photo of the dual (differential) delay line. One delay line is dedicated to the sensing operation, whereas the other is used as a phase and magnitude reference.

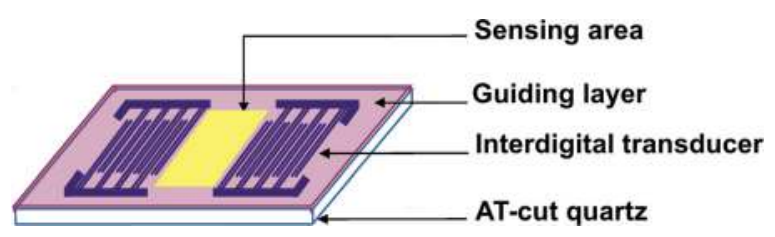

FIG. 1. (Color online) Scheme of the Love-wave based delay line.

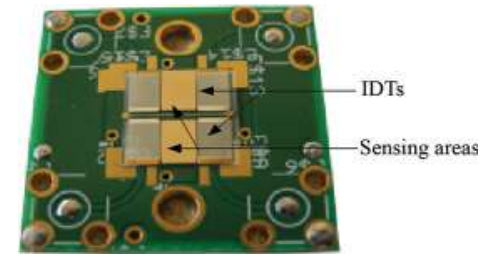

FIG. 2. (Color online) Photo of the dual delay line device glued onto a FR4 epoxy printed circuit $\left(26 \times 26 \mathrm{~mm}^{2}\right)$.

Sensor characterization experiments were done with a Rohde \& Schwartz network analyzer. Figure 3 shows the typical response of silica based Love-wave delay lines. The insertion loss is observed near $-24 \mathrm{~dB}$.

\section{B. Low frequency vibrating mixer}

The low frequency vibrating mixer is an acoustic transducer consisting of an active piezoelectric element and a silicon structure. $^{27}$ First, $100 \mu$ m-thick membranes are structured from a $500 \mu \mathrm{m}$-thick silicon wafer by etching in aqueous potassium hydroxide. A second etching $(50 \mu \mathrm{m})$ step structures a matrix of $5 \times 5$ wells on the top layer in order to receive and easily position $5 \times 5$ droplets. After the drying process, we obtain a $12 \mathrm{~mm} \times 12 \mathrm{~mm} \times 50 \mu \mathrm{m}$ square membrane surrounded by a $25 \mathrm{~mm} \times 30 \mathrm{~mm} \times 450 \mu \mathrm{m}$ rectangular substrate. Then, a massive $10 \mathrm{~mm} \times 10 \mathrm{~mm} \times 127$ $\mu \mathrm{m}$ piezoelectric ceramic is centered and glued to the membrane bottom side using EPOTEK E205 conductive epoxy heated at $80^{\circ} \mathrm{C} .{ }^{9}$ The obtained assembly is then mounted on a printed circuit board. Electrical connections are performed using the ball bonding technique. The use of a $\mathrm{Si}$ structure allows for the choice of the vibration mode. Since our objective in this studyis to prove a concept, we will experiment with the case of only one droplet instead of a $5 \times 5$ droplet matrix. Hence, we choose to excite the acoustic mixer at a degenerated vibrational mode corresponding to a combination of $(1,3)$ and $(3,1)$ modes.

At the resonance frequency, radiation pressure is generated in the droplet, inducing hydrodynamic flow and thus
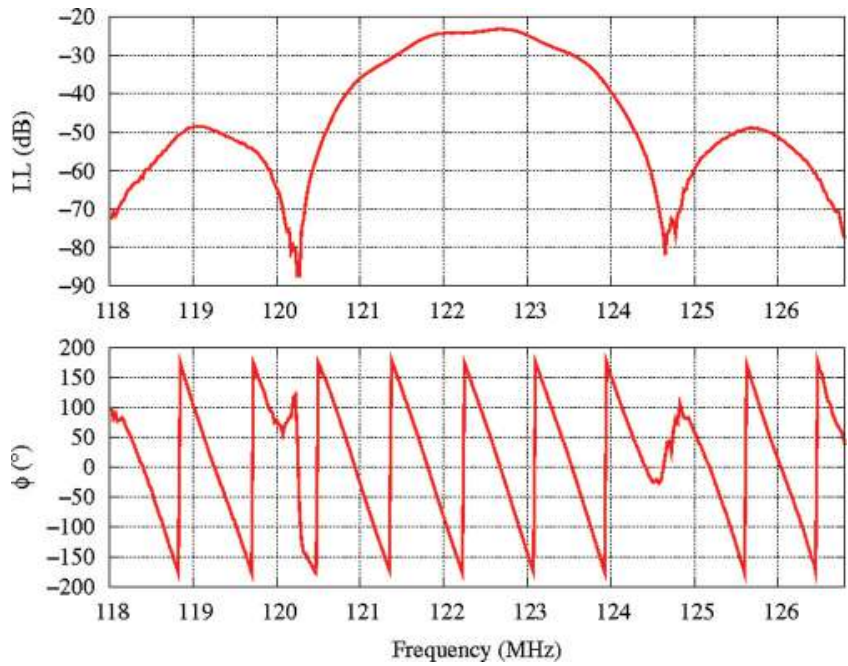

FIG. 3. (Color online) Typical transfer function of delay line exploiting silica guiding overlays (top: insertion loss, bottom: phase). 
TABLE I. Mixer electrical excitation parameters.

\begin{tabular}{lccc}
\hline \hline Start frequency & $63 \mathrm{kHz}$ & Sweep & $10 \mathrm{~ms}$ \\
\hline Stop frequency & $65 \mathrm{kHz}$ & Hold & $0 \mathrm{~ms}$ \\
Signal generator voltage & $55 \mathrm{mV}$ & Return & $1 \mathrm{~ms}$ \\
Offset & $0 \mathrm{mV}$ & Type & Linear \\
Amplified voltage PP & $25 \mathrm{~V}$ & Interval & $1 \mathrm{~ms}$ \\
\hline \hline
\end{tabular}

mixing. Eventually, particles introduced in the droplet will follow flow lines from the drop center to the surface. The particle velocity increases with the active element excitation voltage. Since the mixer resonance frequency depends on the droplet volume and this later changes as a function of time due to evaporation phenomena, the resonance frequency changes over time. To ensure that it remains excited during the whole experiment, the transducer will be excited in a sweep mode around the initial resonance frequency of 63.9 $\mathrm{kHz}$, corresponding to the resonance frequency of the membrane coupled with a $10 \mu \mathrm{L}$ droplet. The excitation parameters are given in Table I.

\section{Biochemical}

The surface functionalization was performed with a selfassembled monolayer of polyethylene glycol O-(2-mercaptoethyl)-O'-(methoxy)-hexaethylene (mPEG thiol) and O-(2carboxiethyl)-O'-(2-mercaptoethyl)-heptaethylene glycol acids (PEG thiol acid) provided by Polypure (Norway). Nhydroxysuccinimide (NHS) and N-(3-dimethylaminopropyl)$\mathrm{N}$-ethylcarbodiimide (EDC) were used as chemical activating groups and were purchased from Biacore (GE Healthcare, Sweden). Water used in the experiments was purified using a water purification system (Purelab prima from ELGA) with a resistivity of $18 \mathrm{M} \Omega \mathrm{cm}$. To study the acoustic excitation influence on the immobilization behavior, we chose to bind the monoclonal antibody $\mathrm{A} 9 \mathrm{H} 12$, at $100 \mu \mathrm{g} \mathrm{mL}^{-1}$ in a $10 \mathrm{mM}$ acetate buffer ( $p \mathrm{H}$ 5.2), which specifically recognizes the Lymphocyte Activation Gene-3 (LAG-3) protein (courteously provided by Immutep SA) (250 nM).

\section{Sensing area functionalization}

The SAW sensor was rinsed with ultrapure ethanol and water. This step was followed by an overnight stay in a mixture of mPEG thiol and PEG thiol acids at $100 \mu \mathrm{M}(7 / 3$ by mole). Those components are diluted in $10 \mathrm{mM}$ acetate buffer $(p \mathrm{H} 4.5)$. The resulting self-assembled monolayer presents, in theory, $30 \%$ by mol of PEG molecules which bear one carboxyl group. The SAW sensor was rinsed with ultrapure water. Then, the two gold sensing areas are activated using NHS at $50 \mathrm{mM}$ and EDC at $400 \mathrm{mM}$ for half an hour in static mode. Afterward, the SAW sensor was rinsed with ultrapure water. This procedure prepares the sensing area for the immobilization step. In this way, covalent immobilization of $\mathrm{Ab}$ could be accomplished via coupling through their primary amines in passive (without agitation) or active (with agitation) modes. The entireprocess is summarized in Fig. 4.

\section{RESULTS AND DISCUSSION}

We now apply our experimental set-up to study the effect of the acoustic mixing on the biological molecule adsorption to the gold sensing area. For that, we propose two experiments. In the first one, we monitor $\mathrm{Ab}$ adsorption on the sensing area in the presence and absence of acoustic mixing. In the second part, we monitor the antigenic reaction of a passively immobilized Ab layer in both modes.

\section{A. Real time detection of Ab binding}

We study the influence of acoustic mixing during the immobilization step on the microarray's behavior. The identification of the binding kinetics parameters requires time resolved $\mathrm{Ab}$ reaction monitoring. To accomplish this, we used a system composed of an acoustic mixer coupled to a Love-wave sensor. The latter is adjusted in the $\mathrm{x}, \mathrm{y}$, and $\mathrm{z}$ axes using micrometric positioners in order to precisely sandwich the drop between the Love-wave device sensing area and the mixer membrane (Fig. 5).

The acoustic properties of the acoustic delay line are monitored at a rate of $1 \mathrm{~Hz}$ using a general purpose interface bus controlled Rohde \& Schwartz ZVC network analyzer. The phase and magnitude of the $S_{21}$ transmission coefficient within the bandpass frequency range are recorded for postprocessing. After its sensing area functionalization, the SAW sensor is rinsed with de-ionized water several times to clean the sensing area before running the adsorption of antibodies.

As a reference, a $10 \mu \mathrm{l}$ droplet of acetate buffer $10 \mathrm{mM}$

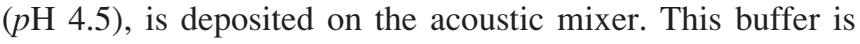

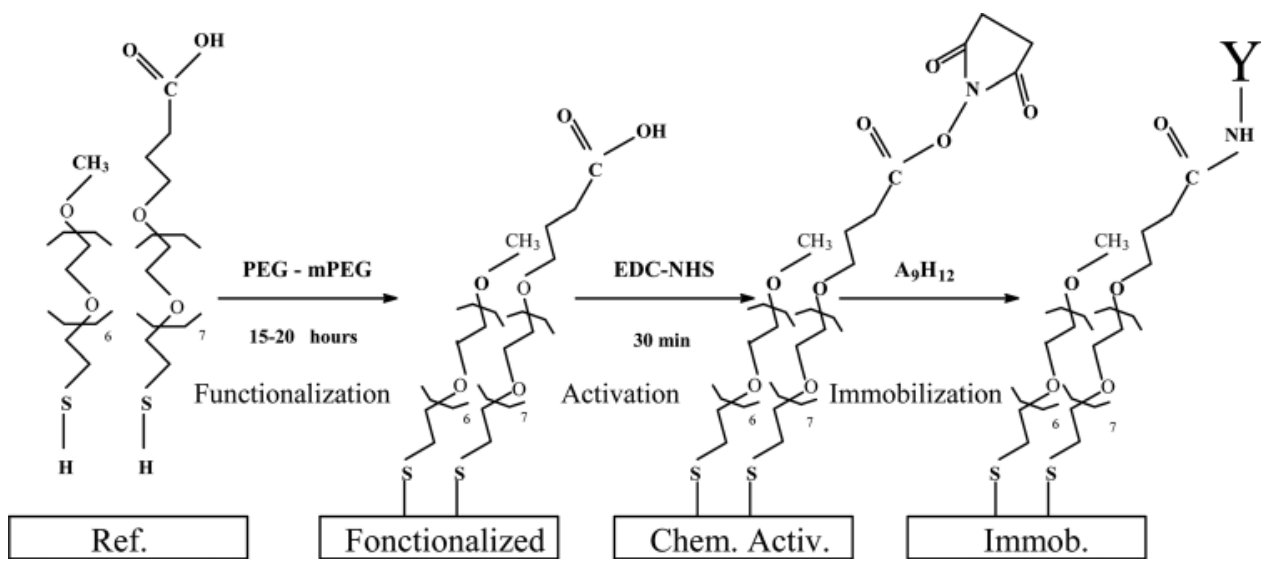

FIG. 4. Chemical functionalization steps; Y represents the antibody. 


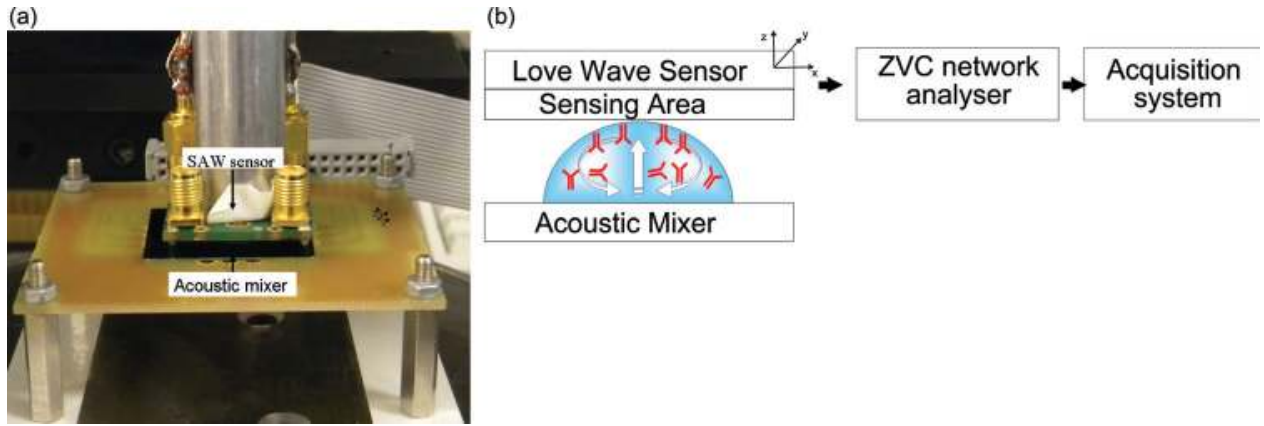

FIG. 5. (Color online) Experimental setup. (a) Love-wave sensor adjusted using micrometer positioner in contact with the acoustic mixer through the droplet; (b) functional scheme of the global setup.

the same used to dilute antibodies. The Love-wave sensor is then adjusted to precisely put the drop in contact with the sensing area while guaranteeing the absence of contact with the IDTs. Once the contact established, the sensor signal is monitored until a stable baseline at about $-27.3 \mathrm{~dB} / 37.7^{\circ}$ is reached (Fig. 6, zone 1). After that, due to the lack of an integrated fluidic system allowing for the continuous flow of various reagents, the SAW sensor and the acoustic mixer are separated in order to rapidly clean the mixer and deposit a new droplet containing antibodies (Fig. 6, zone 2). As soon as the contact is ensured, a shift of the phase and an insertion loss caused by the second droplet filling indicates the beginning of an Ab-film formation at the sensitive surface (Fig. 6, zone 3). If a washing droplet of buffer solution is used the classic cleaning step (denoted by 4 and 5 in Fig. 6), the acoustic phase signal remains stable which demonstrates the binding quality.

Since the dual delay lines of the SAW sensor are identical and were subjected to the same chemical functionalization procedure, the two obtained sensing areas are the same. This fact allows for a true comparison between the active and passive modes of the $\mathrm{Ab}$ binding. Figure 7 exhibits the sensor phase variation in both modes; in the presence of acoustic mixing (bottom curves, associated with the largest phase shift) and in the absence of active mixing (top curves, associated with a lower phase shift). As we can see in this figure, the mixing increases $\mathrm{Ab}$ transfer to the sensing area. In fact, we observe a phase variation of about $8.2^{\circ} \pm 0.3^{\circ}$ in the acoustically activated mode versus $3.3^{\circ} \pm 0.3^{\circ}$ in the passive mode.

Consequently, the noted coverage increase due to mixing is about 2.5. This value is consistent with the mean gain obtained due to the acoustic mixing measured using the surface plasmon resonance imaging $\left(\mathrm{SPRi}^{28}\right)$ technique which is about 2.6 (Ref. 29): in the experiment reported in this reference, we studied the $\mathrm{Ab}$ microarrays optical response to antigenic solutions using a SPRi system. Ab microarrays were built in active and passive modes depending upon whether or not the transducer is excited. They were then integrated to the SPRi apparatus in order to characterize their response to antigenic solutions. This experiment showed that antibodies immobilized in an active way via acoustic mixing better corresponds to antigenic solutions than passively built microarrays with a mean factor of about 2.6. ${ }^{29}$
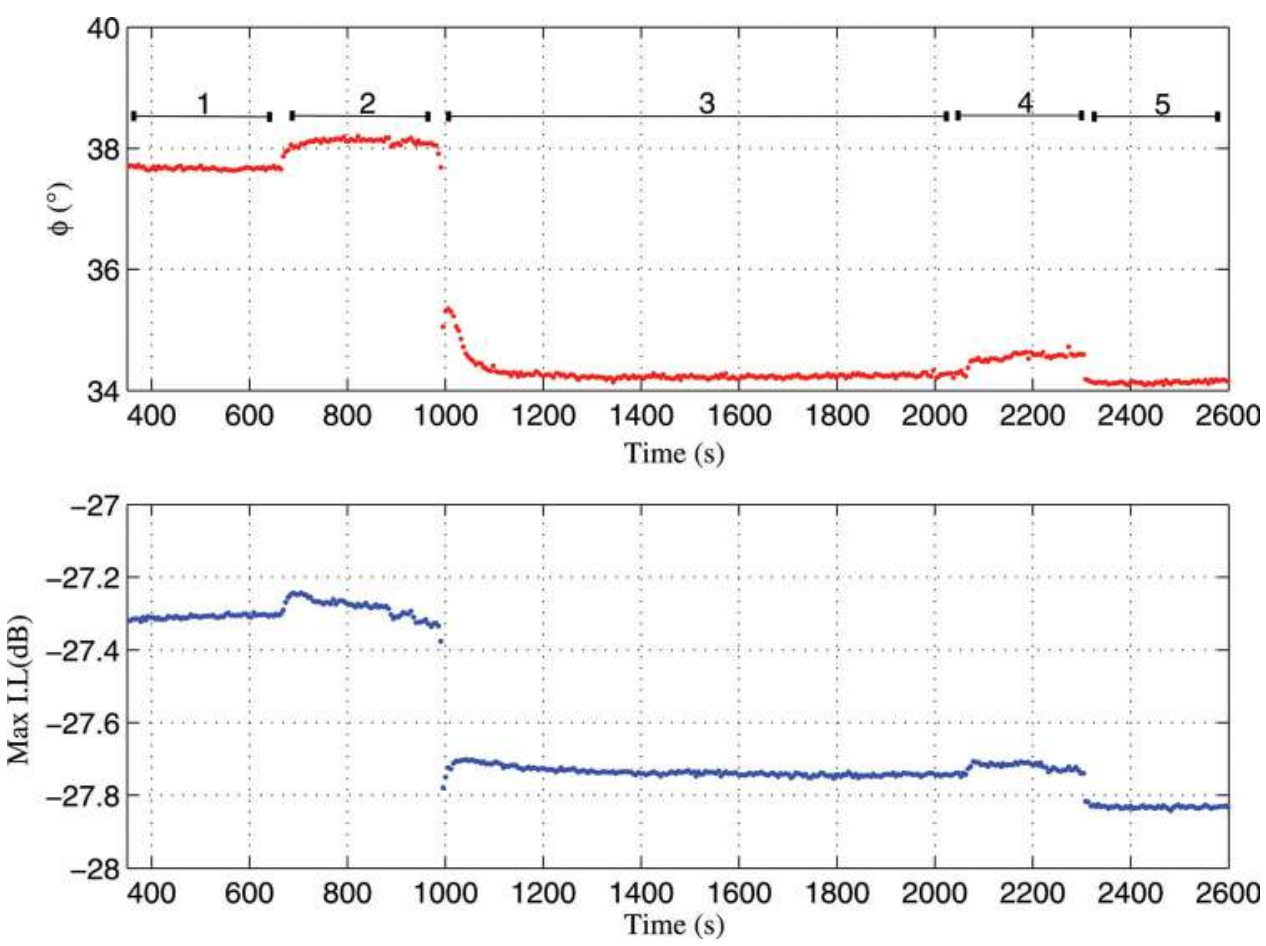

FIG. 6. (Color online) Phase (top) and magnitude (bottom) measurements monitored during $\mathrm{Ab}$ immobilization. 


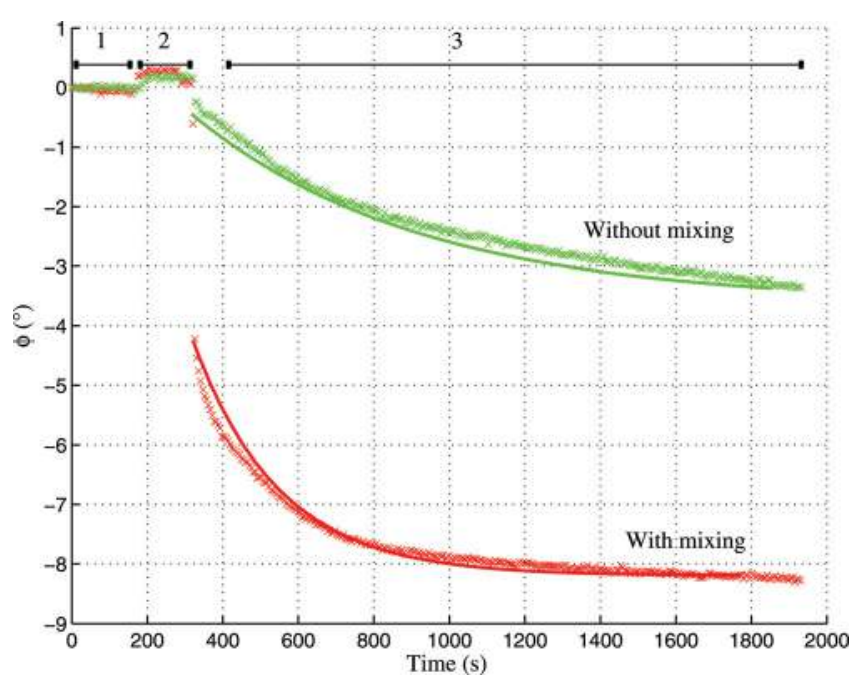

FIG. 7. (Color online) Phase measurements monitored during Ab adsorption for two modes without acoustic mixing (top curves), and with acoustic mixing (bottom curves). The curves are referred to zero at time $t=0$ to make the comparison easier. Crosses are experimental data and solid lines correspond to curve fits following a first order diffusion law.

\section{B. Analysis of acoustic mixing impact on Ab grafting}

Besides the increased coverage, Fig. 7 shows a change in reaction kinetics. To highlight this effect, we fit the $\mathrm{Ab}$ immobilization curves (Fig. 7, time $>327$ s) with a classic first order law [Eq. (1), usually adopted by SPR techniques for kinetics parameters identification ${ }^{30}$

$$
\Phi(t)=K\left[1-\exp \left(\frac{-t}{\tau}\right)\right]
$$

Where $t$ is time, $\tau$ is time constant and $K$ is a constant depending upon concentration.

In the passive case, $\tau$ is equal to 582 s. This time constant is reduced to $280 \mathrm{~s}$ by the acoustic mixing of the droplet. This proves that the acoustic energy makes the reaction faster. Nevertheless, we note that the experimental data are not well described with a single exponential manner; especially in the acoustically activated case, where the first two measured points are not taken into account. In this latter case, antibodies immobilization clearly involves two distinct rate processes and will be better described by Eq. (2)

$$
\Phi(t)=K_{1}\left[1-\exp \left(\frac{-t}{\tau_{1}}\right)\right]+K_{2}\left[1-\exp \left(\frac{-t}{\tau_{2}}\right)\right] .
$$

Indeed, in the absence of acoustic energy, the biological particles are driven to the biochip surface by diffusion. In the acoustically activated case, we believe that particle drift is due to the combination of the induced displacement force and the natural diffusion except inthe vicinity of the biochip where the diffusion is dominant.

When only diffusion acts on particles, the governing law is the Fick second law which is the 1D formulation given by Eq. (3). ${ }^{31}$ The $\mathrm{x}$ axis is taken perpendicular to the sensor surface and parallel to the concentration gradient

$$
\frac{\delta c}{\delta t}=D_{i} \frac{\delta^{2} c}{\delta x^{2}}
$$

If we consider that a quantity of molecules $Q$ is coated as a thin film in an infinite liquid (which practically means that the molecule film thickness is negligible with respect to the liquid height), the concentration of molecules along the liquid is given by the following equation ${ }^{32}$

$$
\frac{c(x, t)}{Q}=\frac{1}{2 \sqrt{\pi D i . t}} \times \exp \left(-\frac{x^{2}}{4 D i . t}\right)
$$

where $x$ is the distance in either direction normal to the initial solute film, $t$ is time, and $D i$ is the diffusion coefficient.

The presented model is only valid as a first approximation since the particles are initially homogeneously spread in the liquid and not plated in a thin film. In order to properly describe the experiment setup, we will suppose that the liquid is an infinite sum of thin molecule films. Each one diffuses fromits initial position, $x_{1}$, in the liquid. Each film contribution is then given by the following relationship

$$
\frac{c(x, t)}{Q}=\frac{1}{2 \sqrt{\pi \text { Di.t }}} \times \exp \left[-\frac{\left(x-x_{1}\right)^{2}}{4 D i . t}\right] .
$$

The molecule quantity $Q_{m}$ that diffused from the $x_{1}$ position and reaches the sensor surface is given by Eq. (6)

$$
Q_{m}\left(x_{1}, t\right)=\int_{X_{\max }}^{\infty} \frac{1}{2 \sqrt{\pi D i . t}} \times \exp \left[-\frac{\left(x-x_{1}\right)^{2}}{4 D i . t}\right] d x .
$$

The total number of molecules that reach the sensor surface, $Q_{\text {tot }}$, at time $t$ is then the sum on the $x_{1}$ position of $Q_{m}$

$$
Q_{\mathrm{tot}}(t)=\int_{0}^{X_{\max }} Q_{m}\left(x_{1}, t\right) d x_{1} .
$$

This relation best fits the experimental measurement in the passive case for a diffusion coefficient $D_{i}$ equal to $10^{-9}$ $\mathrm{m}^{2} / \mathrm{s}$, which is an acceptable value considering that diffusion coefficients of proteins in a liquid vary between $10^{-10}$ and $10^{-9} \mathrm{~m}^{2} / \mathrm{s}$. $^{33}$ Figure 8 shows a similar behavior for the two curves.

In the acoustically activated case, we consider the liquid as two distinguishable domains. The first one extends from the mixer surface, corresponding to $x=0$, to an arbitrary position $x_{D D \rightarrow D}$. In this phase, the particle movement is governed by both diffusion and acoustic flow lines. The second domain goes from the arbitrarily taken interface at $x_{D D \rightarrow D}$ to the SAW sensor surface, corresponding to $x=X_{\max }$. To analytically limit the displacement force influence to the first domain, we consider the function given by the following system

$$
0.5 \times \operatorname{erfc}\left[10^{5}\left(x_{1}-x_{D D \rightarrow D}\right)\right]=\left\{\begin{array}{ll}
1 & \text { if } x<x_{D D \rightarrow D} \\
0 & \text { if } x>x_{D D \rightarrow D}
\end{array},\right.
$$

where erfc is the complementary error function.

The considered solution of the Fick second law is consequently modified by a mean velocity $\langle\mathrm{v}\rangle$ which demonstrates 
(a)

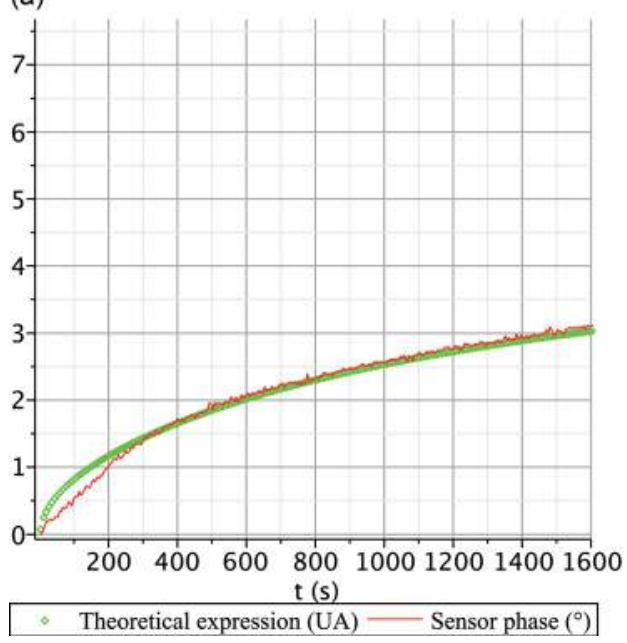

(b)

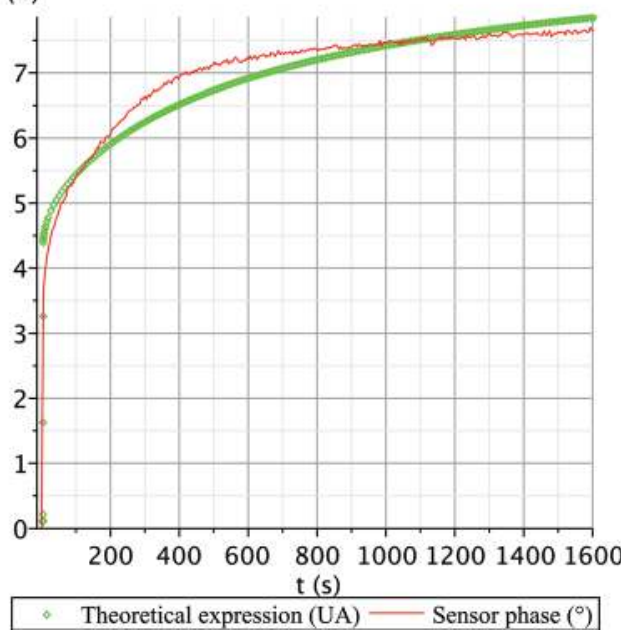

FIG. 8. (Color online) Comparison of normalized phase measurements monitored during $\mathrm{Ab}$ adsorption (thin solid lines) with theoretical laws (thick solid lines) in two modes: (a) without acoustic mixing and (b) with acoustic mixing. the presence of a displacement force. This magnitude is taken equal to $1 \mathrm{~mm} / \mathrm{s}$ which is an experimentally determined value (data not shown)

$$
\begin{aligned}
& \frac{c(x, t)}{Q}=\frac{1}{2 \sqrt{\pi D i . t}} \exp \\
& \times\left(-\frac{\left\{x-x_{1}-\langle v\rangle \times t \times 0.5 \times \operatorname{erfc}\left[10^{5}\left(x_{1}-x_{D D \rightarrow D}\right)\right]\right\}^{2}}{4 D i . t}\right) .
\end{aligned}
$$

For an $x_{D D \rightarrow D}$ equal to $0.7 \mathrm{~mm}$, the expression given by Eq. 10, shows a behavior similar to the experimental data in the active case

$$
\begin{aligned}
& \frac{c(x, t)}{Q}=\int_{0}^{X_{\max }} \int_{X_{\max }}^{\infty} \frac{1}{2 \sqrt{\pi D i . t}} \exp \\
& \times\left(-\frac{\left\{x-x_{1}-\langle v\rangle \times t \times 0.5 \times \operatorname{erfc}\left[10^{5}\left(x_{1}-x_{D D \rightarrow D}\right)\right]\right\}^{2}}{4 \text { Di.t }}\right) d x d x_{1} .
\end{aligned}
$$

In both passive and active cases, the magnitude of the theoretical expression is not significant, since we focus on the diffusion coefficient identification by using the normalized adsorption curves. In particular, the correspondence of theoretical and experimental time constants, as illustrated in Fig. 8, demonstrates the accuracy of our approach. Nevertheless, the presented equations are a $1 \mathrm{D}$ formulation of the real problem in order to make the calculation easier. A 3D formulation would better approach the experimental data.

The immobilized quantity over the sensing area is deduced from the combination of two equations. The phasefrequency slope is given by the acoustic wave velocity, considering that one wavelength propagation is equal to a phase rotation of $360^{\circ}$, thus

$$
\frac{\Delta \phi}{\Delta f}=\frac{360 . L}{V}=3.7 \times 10^{-4}(\mathrm{deg} / \mathrm{Hz})
$$

where $V=5000 \mathrm{~m} \mathrm{~s}^{-1}$ is the phase velocity and $L=5.23$ $\mathrm{mm}$, is the center-to-center distance between the IDTs.

Alternatively, the mass sensitivity $S$ for the acoustic wave sensors is defined as the incremental frequency change occurring in response to an incremental change in mass per unit of area $A$ on the surface of the device as follows

$$
S=\frac{\Delta f}{f_{0}} \frac{A}{\Delta m}\left(\mathrm{~cm}^{2} / \mathrm{g}\right)
$$

where $\Delta m$ is the uniformly distributed mass added to the surface of the device, $f_{0}(125 \mathrm{MHz})$ is the unperturbed resonance frequency of the device, and $\Delta f$ is the change in the operational frequency due to the mass loading effect. The gravimetric sensitivity was measured in liquid phase and is equal, for these sensors, to $250 \mathrm{~cm}^{2} / \mathrm{g}$. ${ }^{34}$ The expression of the surface density [Eq. (4)] follows from Eqs. (11) and (12).

$$
\frac{\Delta m}{A}=\frac{\Delta \Phi}{f_{0} \cdot S \times 3.7 \times 10^{-4}}\left(\mathrm{~g} / \mathrm{cm}^{2}\right) .
$$

The phase shift corresponding to the active and passive modes are, respectively, $8.2^{\circ} \pm 0.3^{\circ}$ and $3.3^{\circ} \pm 0.3^{\circ}$ (Fig. 7) and the absorbed mass are, respectively, $713 \mathrm{ng} / \mathrm{cm}^{2}$ and 290 $\mathrm{ng} / \mathrm{cm}^{2}$, using Eq. (13).

\section{Experimental assessment of the acoustic mixing influence on $\mathrm{Ab}-\mathrm{Ag}$ reaction}

Since we have observed an important improvement of the antibody immobilization due to acoustic mixing, we have investigated the influence of this energy on the immobilized layer response to the antigenic solution. To do that, we have passively $\mathrm{Ab}$ immobilized the two sensing areas of the sensor. In fact, an Ab containing droplet was deposited on each sensing area during $30 \mathrm{~min}$. The sensor was subsequently incubated in ethanolamine (1 M) to target free NHS entities in order to deactivate the sensing areas. It wasthen rinsed with ultrapure water. Our previous measurements allow an estimation of the $\mathrm{Ab}$ surface density to be about $300 \mathrm{ng} / \mathrm{cm}^{2}$ (see Sec. 2.1). The sensor is now prepared to begin acquisition 


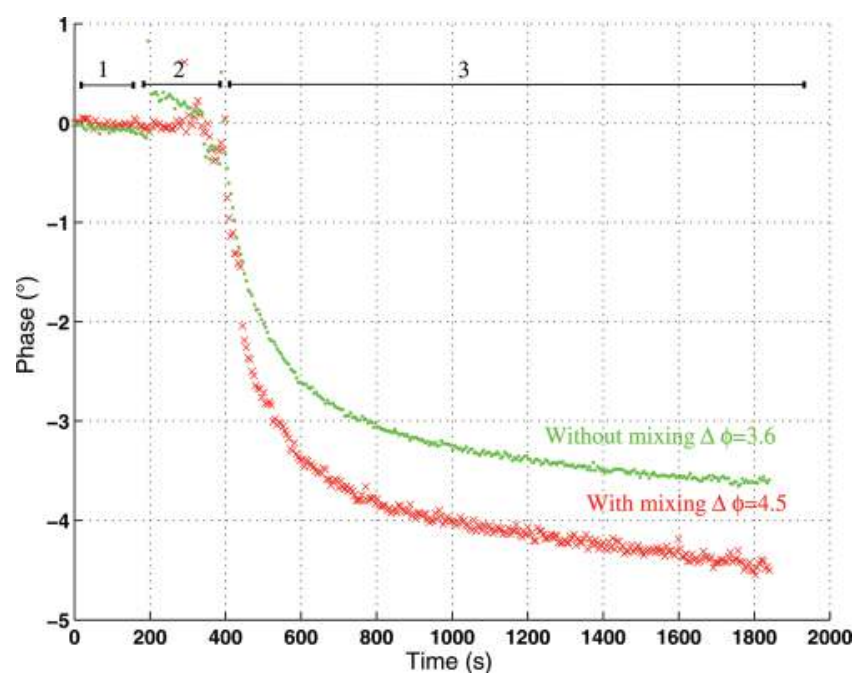

FIG. 9. (Color online) Monitored phase measurements of the Ab layer response toan antigenic solution for two modes without acoustic mixing (circles, top curve), and with acoustic mixing (crosses, bottom curve). The curves are referred to zero at time 0 to make comparison easier.

(Fig. 9). We proceed, as in the Ab immobilization monitoring, by first takinga baseline reference (zone 1). In this case, the reference is the antigen buffer PBS. After the cleaning step (referred to as 2 in Fig. 9), a $10 \mu \mathrm{L}$ droplet of antigenic solution is deposited on the acoustic mixer membrane. Since the contact is ensured, the Ab-Ag reaction begins (Fig. 9, zone 3). We have realized this experiment in both active and passive modes. Without acoustic mixing during the antigenic reaction, the phase shifts with $3.6^{\circ} \pm 0.3^{\circ}$, which corresponds to an $\mathrm{Ag}$ surface density of $311 \mathrm{ng} / \mathrm{cm}^{2}$. This is consistent with previously established SPR experiments (data not shown) exhibiting saturating levels at a molar ratio of $1 / 1$ (when $50 \%$ of Fab sites are occupied) for this $\mathrm{Ab} / \mathrm{Ag}$ couple. This indicates that the expected Ag surface density is equal to the one of the Ab layers, $300 \mathrm{ng} / \mathrm{cm}^{2}$. The antigenic response in the acoustically excited case induces a phase shift of about $4.5^{\circ} \pm 0.3^{\circ}$, corresponding to $389 \mathrm{ng} / \mathrm{cm}^{2}$ surface density. The global increase compared to the passive case is about $20 \%$. This indicates that acoustic mixing allowed the antigens to find a way into the classically unbound Fab sites.

\section{CONCLUSIONS}

We propose a Love-wave sensor whose phase shifts as a function of the immobilized Ab quantity, combined with an active acoustic mixing device. We demonstrate its use during the immobilization step for improved coverage while keeping the thermal effect below detectable limits.

We have assessed that mixing at the droplet level increases antibodies $(\mathrm{Ab})$ transfer to a sensing area surface, increases the reaction kinetics by removing the dependency with the protein diffusion coefficient in a liquid, while inducing minimum disturbance to the sensing capability of the Love mode. We have tested the global system composed of the acoustic mixer coupled to the SAW sensor. In this way, we proved that the $\mathrm{Ab}$ density on the sensing surface is improved by acoustic mixing with a gain factor of about 2.5. Beyond the asymptotic transfer rate, the time dependent kinetic modeling yields a protein diffu- sion coefficient consistent with the literature in the case of the static drop, and an increased transfer rate dependent upon the fluid velocity in the case of acoustic mixing. Typical fluid velocities in the $\mathrm{mm} / \mathrm{s}$ range included in the Fick diffusion law yields the best fit of the experimental data.

This experiment also showed an improvement of the captured Ag density of $20 \%$ compared to the passive antigenic interaction.

\section{ACKNOWLEDGMENTS}

The authors would like to thank Benoît Simon and Alain Rouleau for their assistance in biological solution preparation. We also thank Dr. Frédéric Triebel (from Immutep SA) for providing the A9H12/LAG-3 model and the clean room and technology platform MIMENTO (Besançon, France).

${ }^{1}$ E. Gizeli and C. R. Lowe, Biomolecular Sensors (Taylor \& Francis, London, 2002).

${ }^{2}$ T. M. A. Gronewold, Anal. Chim. Acta 603, 119 (2007).

${ }^{3}$ B. Jakoby and M. Vellekoop, Sens. Actuators, A 68, 275 (1998).

${ }^{4}$ L. El Fissi, J.-M. Friedt, V. Luzet, F. Cherioux, G. Martin, and S. Ballandras, IEEE Freq. Control Symp. 861, 2009.

${ }^{5}$ L. El Fissi, J.-M. Friedt, F. Chérioux, and S. Ballandras, Sens. Actuators, B 144, 23 (2010).

${ }^{6}$ C. Modin, A.-L. Stranne, M. Foss, M. Duch, J. Justesen, J. Chevallier, L. K. Andersen, A. G. Hemmersam, F. S. Pedersen, and F. Besenbacher, Biomaterials 27, 1346 (2006)

${ }^{7}$ C. Kößlinger, E. Uttenthaler, S. Drost, F. Aberl, H. Wolf, G. Brink, A. Stanglmaier, and E. Sackmann, Sens. Actuators,. B 24, 107 (1995).

${ }^{8}$ J. Malmström, H. Agheli, P. Kingshott, and D. S. Sutherland, Langmuir 23, 9760 (2007).

${ }^{9}$ M. Wilczewski, A. Van der Heyden, O. Renaudet, P. Dumy, L. CocheGuerente, and P. Labbe, Org. Biomol. Chem. 6, 1114 (2008).

${ }^{10}$ F. Bender, P. Roach, A. Tsortos, G. Papadakis, M. I. Newton, G. McHale, and E. Gizeli, Meas. Sci. Technol. 20, 124011 (2009).

${ }^{11}$ T. Mangeat, A. Berthier, C. E. Caille, M. Perrin, W. Boireau, C. Pieralli, and B. Wacogne, Laser Phys. 19, 252(2009).

${ }^{12}$ K. Mitsakakis, A. Tserepi, and E. Gizeli, Microelectron. Eng. 86, 1416 (2009).

${ }^{13}$ K. Mitsakakis, A. Tserepi, and E. Gizeli, J. Microelectromech. Syst. 17(4), 1010 (2008).

${ }^{14}$ G. Ohlsson, P. Axelsson, J. Henry, S. Petronis, S. Svedhem, and B. Kasemo, Microfluid. Nanofluid. 9, 705 (2010).

${ }^{15}$ B. Liedberg, C. Nylander, and I. Lundstrom, Biosens. Bioelectron. 10, 1 (1995).

${ }^{16}$ W. Boireau, A. Rouleau, G. Lucchi, and P. Ducoroy, Biosens. Bioelectron. 24, 1121 (2009).

${ }^{17}$ R. Karlsson, H. Roos, L. Fagerstam, and B. Persson, Methods 6, 99 (1994).

${ }^{18} \mathrm{P}$. Tabeling, Introduction to Microfluidics (Oxford University Press, London, 2005).

${ }^{19}$ T. M. Squires and S. R. Quake, Rev. Mod. Phys. 7, 977 (2005).

${ }^{20}$ M. K. Tan, J. R. Friend, and L. Y. Yeo, 16th Australian Fluid Mechanics Conference, Gold Coast, Queensland, Australia, 3-7, December 2007, pp. 790-793.

${ }^{21}$ H. Song, M. R. Bringer, J. D. Tice, C. J. Gerdts, and R. F. Ismagilov, Appl. Phys. Lett. 83, 4664 (2003).

${ }^{22}$ P. Paik, V. K. Pamula, M. G. Pollack, and R. B. Fair, Lab Chip 3, 28 (2003).

${ }^{23}$ A. Lee, A. Lemoff, and R. Miles, US Patent No. US6733172 (2004).

${ }^{24}$ E. Galopin, M. Beaugeois, B. Pinchemel, J.-C. Camart, M. Bouazaoui, and V. Thomy, Biosens. Bioelectron. 23, 746 (2007).

${ }^{25}$ A. Renaudin, V. Chabot, E. Grondin, V. Aimez, and P. G. Charrette, Lab Chip 10, 111 (2010).

${ }^{26}$ L. El Fissi, "Detection and measurement of nanoparticles for the sensors applications in liquid medium," Ph.D. dissertation, University of FrancheComte in Besançon (France), 2009, [in French].

${ }^{27}$ F. Kardous, R. Yahiaoui, and J.-F. Manceau, IEEE International Ultrasonics Symposium Proceedings, 2533-2536, 2009.

${ }^{28}$ B. P. Corgier, S. Bellon, M. Anger-Leroy, L. J. Blum, and C. A. Marquette, Langmuir, 25, 9619 (2009) 
${ }^{29}$ F. Kardous, A. Rouleau, B. Simon, R. Yahiaoui, J.-F. Manceau, and W. Boireau, Biosens. Bioelectron. 26, 1666 (2010).

${ }^{30} \mathrm{E}$. Maillart, "Développement d'un système optique d'imagerie en résonance de plasmons de surface pour l'analyse simultanée de multiples interactions biomoléculaires en temps reel," Ph.D. dissertation, University of Paris XI (France), 2004, [in French].
${ }^{31}$ J. Crank, The Mathematics of Diffusion (Oxford University Press, London, 1956). ${ }^{32}$ H. B. Fischer, J. E. List, C. R. Koh, J. Imberger, and N. H. Brooks, Mixing in Inland and Coastal Waters (Academic, New York, 1979).

${ }^{33}$ D. Brune and S. Kim, PNAS 90, 3835 (1993).

${ }^{34}$ J.-M. Friedt, K. H. Choi, F. Frederix, and A. Campitelli, J. Electrochem. Soc. 150, 229 (2003). 